\title{
Risk assessment of a bulk cryogenic tank: beyond the leak before break criterion
}

\author{
M. Compare \& L. Legnani \\ Aramis Srl, Milano, Italy \\ G. Sansavini \\ ETHZ, Zurich, Switzerland
}

M. Buldrini

Nier Ingegneria, Bologna, Italy

E. Zio

Ecole Centrale Paris and Supelec, Paris, France \& Politecnico di Milano, Milano, Italy

L. Beretta

SOL Group, Monza, Italy

ABSTRACT: The increase in the size and production capacity of air separation plants has boosted the need of developing methodologies to properly assess the risk related to major releases of liquefied gas. In this respect, the leak-before-break (LBB) assessment is currently adopted to demonstrate the safety of the structures containing liquefied gas, under the assumption that the tank is always operated in nominal conditions. This assumption is questioned in this paper, which proposes a new methodology for the assessment of the risks related to cryogenic tank catastrophic rupture. The methodology provides a comprehensive understanding of the issues associated to the worst case rupture scenario: from the investigation of the causes of the undesirable operating conditions up to the analysis of the associated structural consequences, within a probabilistic framework.

\section{INTRODUCTION}

The increase in the size and production capacity of air separation plants, has led to a growing capacity of cryogenic liquid storage installations at production sites. Therefore, considering the potential hazards associated with liquid products has become a fundamental issue to address, together with the estimation of the effects of a major release of liquid on the local environment, and the development of preventive measures.

Nowadays, evaluating the structural safety of the cryogenic tanks by critically examining the integrity of new or existing constructions is a common practice. To do this, non-destructive testing methods for flaws detection are usually performed, whereas acceptability criteria for the revealed flaws have been defined in standards of the sector. In this respect, a typical approach to demonstrate the safety of a structure containing defects is to design it under the leakbefore-break (LBB) criterion; the scope is to guarantee that in nominal operating conditions the stress conditions inside the structure are such that the growth of flaws leaves time for a stable leak of the liquefied gas preceding the occurrence of a sudden disruptive break of the structure. Thus, a leakage of fluid through a crack can be detected before it reaches a critical dimension; this allows preventing catastrophic liquefied gas releases.

In practice, the LBB design criterion is based on setting two critical values for the crack length: $l_{C}$ and $l_{L}$. The former represents the maximum length that prevents the tank from experiencing a catastrophic rupture; the latter represents the minimum length to have a fluid leakage. To meet the LBB criterion, the designers have to prove that $l_{L}<l_{C}$ with a suitable margin: in this case, a fluid leakage is detected before the catastrophic failure. In particular, the analysis is conducted at the most risky vessel sections, i.e, the sections with extreme loading conditions and poorest material properties.

However, the traditional LBB approach suffers from the limitation of considering the tank behavior in nominal operating conditions, only. Indeed, it is also interesting to analyze the worst case rupture scenario, which, for the structure under study, coincides with the tank catastrophic rupture, caused by potential malfunctions of the control system or the safety devices.

In this context, the paper proposes a new approach to the assessment of the risks related to the catastrophic rupture of a flat bottom cryogenic tank containing Liquefied Oxygen (LOX). The rupture is considered catastrophic if it leads to an uncontrollable nearly-instantaneous release of the entire liquefied gas content. The assessment aims at reaching a comprehensive understanding of the problems related to the tank rupture, from the investigation of the causes of abnormal operating conditions up to the analysis of the associated structural consequences, within a probabilistic framework.

The proposed procedure consists of two parts. In the first part, Failure Mode and Effect Analysis (FMEA) and Fault Tree Analysis (FTA) guide the 
investigation of the storage system in order to identify the critical events that may evolve in dangerous scenarios affecting the structural safety of the tank, together with the associated occurrence frequencies. This part of the study also includes sensitivity and uncertainty analysis, which allow improving the robustness of the results. In the second part, structural analyses based on Finite Element Methods (FEM) are performed to investigate the behavior of the tank in both ordinary and abnormal operating conditions. In particular, the abnormal conditions are those in which the vessel is under exceptional stresses, due to the critical scenarios identified by FMEA and FTA. The combination of FMEA, FTA and FEM structural analyses allows identifying and assessing the stress conditions which may lead to a catastrophic rupture of the tank. The main outcome of such an analysis is a list of recommendations such as the installation of protective equipment and instrumentation, definition of a strategy for equipment inspection and testing, identification of the components for which a re-design may be convenient, etc. This allows reducing the occurrence of large releases of cryogenic fluids, by providing useful guidelines to the engineers directly involved in the design, installation, operation and maintenance of bulk cryogenic liquid storage systems.

\section{CASE STUDY}

The methodology is illustrated by way of a real case study concerning a LOX flat bottom cryogenic tank, owned by SOL Group and sited in Frankfurt. The analysis focuses on the cryogenic tank and all the pieces of equipment connected to it. This is compliant to the definition of tank given by EIGA IGC Doc. 127/13/E(EIGA 2013):

"the total fixed assembly of liquid storage $\operatorname{tank}(\mathrm{s})$ integrated with other equipment, such as pumps, filling equipment, pressure build-up vaporizers, controls and other related ancillary equipment that are connected to it."

The cryogenic tank is made up of two different structures: the internal vessel, which is designed to contain the cryogenic liquid, and the outer casing, which contains the insulating material that ensures a correct storage of the liquefied gas. The inner tank, with capacity of $2000 \mathrm{~m}^{3}$, is made of stainless steel A240-304; this material is capable of withstanding cryogenic temperatures of the liquefied oxygen, which is stored at $-183{ }^{\circ} \mathrm{C}$, at nearly atmospheric pressure $(-0.0022$ bar $<\mathrm{P}<0.095$ bar $)$. The outer casing, made of $\mathrm{A} 283 \mathrm{grC}$, is not designed to withstand low temperatures, as it is not wetted by liquefied oxygen in normal operations.

Both vessels are connected to a number of components which allow performing the liquefied oxygen load and unload operations, and guarantee that the operating pressure, temperature and level conditions are close to the corresponding design values.

The tank is usually filled with the liquefied oxygen from the liquefaction unit, although sometimes it can be stocked up with liquid from tanker track. The unloading operation is performed by means of cryogenic pumps, which fill the tanker tracks for final distribution. A drain system is also installed on the inner vessel to allow the complete removal of the stored liquid in emergency conditions.

The annular space delimited between the inner and outer vessels is intended to ensure a thermal insulation. To this aim, it is filled up with perlite, an amorphous volcanic glass with high insulating characteristics and light weight. Moreover, to guarantee a better insulation quality, the annular space is also continuously flushed with nitrogen, which allows keeping the insulation in a dry state. The nitrogen flow is provided by nozzles located at the inner vessel bottom, and is released in proximity of the top of the tank.

Table 1 and Table 2 summarize the material properties and the operating conditions of the inner vessel and the annular space, respectively.

Table 1. Material properties.

\begin{tabular}{lll}
\hline & Oxygen & Nitrogen \\
\hline Content in air & $21 \mathrm{Vol} \%$ & $78.1 \mathrm{Vol} \%$ \\
Gas density $\left(1.013\right.$ bar, $\left.15^{\circ} \mathrm{C}\right)$ & $1.36 \mathrm{~kg} / \mathrm{m}^{3}$ & $1.19 \mathrm{~kg} / \mathrm{m}^{3}$ \\
Boiling temperature $(1.013 \mathrm{bar})$ & $-183{ }^{\circ} \mathrm{C}$ & $-196{ }^{\circ} \mathrm{C}$ \\
Liquid density $(1.013$ bar, boil T) & $1.14 \mathrm{~kg} / 1$ & $0.8 \mathrm{~kg} / \mathrm{l}$ \\
\hline
\end{tabular}

Table 2. Operating conditions for the LOX storage system.

\begin{tabular}{llc}
\hline & $\begin{array}{c}\text { Inner tank } \\
\text { Oxygen }\end{array}$ & $\begin{array}{c}\text { Annular space } \\
\text { Nitrogen }\end{array}$ \\
\hline Temperature & $-183{ }^{\circ} \mathrm{C}$ & $+20{ }^{\circ} \mathrm{C}$ \\
Vapor space press max & $+0.095 \mathrm{bar}$ & $+0.01 \mathrm{bar}$ \\
Max negative pressure & $-0.0022 \mathrm{bar}$ & $-0.0022 \mathrm{bar}$ \\
Max level & $14.32 \mathrm{~m}$ & \\
\hline
\end{tabular}

The operating conditions are controlled by two redundant systems: an equipment installed to regulate the value of the parameters during normal operations, and a set of safety devices to prevent the occurrence of dangerous conditions in the tanks, when regulating system fails. In addition, the vessels are provided with detectors and alarms, which go off when measured parameters assume unexpected values.

\section{A NOVEL APPROACH TO RISK ASSESSMENT OF A CRYOGENIC TANK}

The risk associated to the catastrophic rupture of the cryogenic storage system described above has been studied by applying a novel and comprehensive ap- 
proach, which can be summarized in the following three steps:

1. FMEA and FTA. These traditional techniques of risk analysis allow identifying the critical events that may lead to dangerous scenarios affecting the structural safety of the tank, and estimating the associated occurrence frequencies.

2. Uncertainty and sensitivity analyses, which are performed to assess the robustness of the results obtained from the previous step.

3. Structural analyses, performed to evaluate the severity of the consequences of the tank rupture both in ordinary operating conditions and in the critical scenarios identified by FMEA and FTA.

In the following Sections, the different steps are detailed, and the corresponding results commented.

\subsection{Identification of critical events dangerous for the structural safety of the cryogenic tank}

\subsubsection{Procedure}

In the first step of the procedure, a FMEA study is performed, whose core is to go over all the potential component failure modes, identify the consequent losses of system functionalities and configure the resulting outcomes from the accidental scenarios; a qualitative evaluation of the criticality of the failure modes is also given by the analysts in terms of severity of consequences and likelihood of occurrence. These two latter attributes, severity and likelihood, are the entries of a criticality matrix, which allows assessing whether the failure modes are critical and violate the safety requirements.

This analysis has supported a full understanding of the system at hand, and its main outcomes have been:

- The functions implemented by the system;

- The items involved in performing these functions;

- The consequence of each loss of function. This information is used for the identification of the most severe events in relation to the tank integrity, to be further investigated;

- The identification of the most critical elements of the system, whose malfunctions can affect the integrity of the tank or the success of functions that concur in assuring the structural integrity;

- The identification of the failure modes and failure rates associated to the critical items;

- The detection means of the different failure modes;

- The redundancies of the system, listed in the fields "compensatory measures" and "remarks".

Once the critical events have been identified, a quantitative FTA has been carried out to estimate the occurrence frequencies of the undesired events.

\subsubsection{Results}

The functioning of the storage system has been analyzed with respect to eight main functions:

1. Inner tank pressure regulation;

2. Inner tank pressure safety control;

3. Inner tank level safety control;

4. Liquid extraction;

5. Liquid introduction;

6. Insulation;

7. Annular space pressure safety control;

8. Oxygen containment.

The FMEA has shown that the safety related functions are the most critical among the eight above. This is an expected result, as safety related functions are involved in mitigating or compensating conditions potentially dangerous for the tank integrity. In this respect, Table 3 shows the critical functions and the subsystems involved in their implementations.

Table 3: Critical functions and related subsystems.

\begin{tabular}{|c|c|}
\hline FUNCTIONS & Associated SUBSYSTEMS \\
\hline $\begin{array}{c}\text { INNER TANK } \\
\text { PRESSURE } \\
\text { SAFETY CONTROL }\end{array}$ & $\begin{array}{l}\text { Pressure Safety Valve PSV4001 } \\
\text { Pressure Safety Valve PSV } 4002\end{array}$ \\
\hline $\begin{array}{c}\text { INNER TANK } \\
\text { LEVEL } \\
\text { SAFETY CONTROL }\end{array}$ & $\begin{array}{l}\text { Differential pressure level meter } \\
\text { LT/LI4002 } \\
\text { Differential pressure level meter } \\
\text { LT/LI4003 } \\
\text { Level switch } \begin{array}{l}\text { LSHH/LSH4004 }\end{array}\end{array}$ \\
\hline $\begin{array}{l}\text { ANNULAR SPACE } \\
\text { PRESSURE } \\
\text { SAFETY CONTROL }\end{array}$ & $\begin{array}{l}\text { Pressure Safety Valve PSV4003 } \\
\text { Pressure Safety Vent } \quad \text { PSE4001 }\end{array}$ \\
\hline
\end{tabular}

Finally, the procedure has allowed to identify four critical events and their occurrence frequencies:

- TOP event 1: overpressure in the inner tank (pressure $>95 \mathrm{mbar}$ ), as consequence of the malfunction of the pressure safety subsystems;

- TOP event 2: under pressure (i.e., vacuum) in the inner tank (pressure $<-2.2 \mathrm{mbar}$ ), as consequence of the malfunction of the pressure safety subsystems;

- TOP event 3: extreme liquid level in the inner tank (level >14.32m), as consequence of the malfunction of the level safety subsystems;

- TOP event 4: overpressure in the annular space (pressure $>10 \mathrm{mbar}$ ), as consequence of the malfunction of the pressure safety subsystems.

The failure frequencies of the four critical events, at time $t=20$ years are listed in Table 4 . In particular, these values refer to two different settings:

1. The Pressure Safety Valves (PSV) installed on the tank to protect the structure against overpres- 
sures are periodically inspected, with a period of 4 years;

2. PSV are not inspected.

In this respect, notice that the regular inspection and maintenance of the pressure safety devices is not mandatory for the typology of tank under analysis, since the particular vessel is not required to comply with the Pressure Equipment Directive (97/23/EC).

The two different work hypotheses yield two values of occurrence frequencies. In fact, the inspection interval enters the quantification of the availability of dormant devices such as the safety valves degradation (Zio 2007). In particular, from Table 3 it emerges that even when the PSV are not periodically inspected, the frequencies of the four top events have very small values (smaller than $10^{-7} \mathrm{y}^{-1}$ ). The small values of these frequencies are due to the fact that long sequences of failure events have to occur to get the top event; in addition, as illustrated in Table 3 , there is redundancy of safety control devices.

On the other side, from Table 4 it also emerges that when the PSV are regularly maintained, then the occurrence frequencies of the top events decrease significantly. Therefore, a periodic inspection of the PSVs is highly recommended for any generic flat bottom cryogenic tank, even if not mandatory.

Table 4. Occurrence frequencies of critical events.

\begin{tabular}{lll}
\hline & \multicolumn{3}{c}{$\begin{array}{c}\text { Failure frequency } \\
\text { events/year }\end{array}$} \\
\hline & PSV inspected & PSV not inspected \\
\hline TOP event 1 & $8.76 \mathrm{e}-12$ & $7.86 \mathrm{e}-10$ \\
TOP event 2 & $3.64 \mathrm{e}-10$ & $3.27 \mathrm{e}-8$ \\
TOP event 3 & $8.30 \mathrm{e}-9$ & $7.93 \mathrm{e}-8$ \\
TOP event 4 & $1.85 \mathrm{e}-10$ & $1.68 \mathrm{e}-8$ \\
\hline
\end{tabular}

\subsection{Robustness of the results}

Importance, uncertainty and sensitivity analyses have been performed in order to investigate the robustness of the results. In particular, these additional analyses have been carried out for every critical event, with a twofold scope:

1. Identifying the component failures or basic events which most influence the event occurrence frequency. This allows to identify the system weaknesses, and provides guidelines for effective actions for system improvements;

2. Estimating the uncertainty on the critical event occurrence frequencies, which comes from the lack of precise knowledge about the failure model parameters.

\subsubsection{Importance analysis}

Generally speaking, Importance Measures (IMs) aim at quantifying the contribution of components or basic events to the considered measure of system performance, which can be the system reliability, unreliability, unavailability or risk, depending on the application (Zio 2007; Baraldi et al. 2009). Information about the importance of the components constituting a system with respect to its safety and availability is of great practical aid: the identification of the components which mostly determine the overall system behaviour allows identifying system vulnerabilities and the most effective actions for the system improvement.

Different IMs have been proposed in literature, which allow investigating different system features. In particular, the Birnbaum IM expresses the contribution to system reliability of the reliability of the various system components (Baraldi et al. 2013). In details, components for which a variation in the reliability results in the largest variation of the system reliability have the highest importance. Thus, a reduction in the occurrence probability of the most important events is expected to result in a reduction of the occurrence probability of the TOP event.

With reference to the analysed case study, the Birnbaum IM have been computed for each basic event constituting the Fault Trees of the four investigated critical events. This has allowed to identify the most important basic events, and thus the improvement actions with the highest priority.

The results of the analysis can be summarized as follows:

- The failure of the inner tank pressure Safety Valves greatly influences two out of four critical events (TOP event 1 and TOP event 2). Then, improving the reliability of these items is expected to lead to a great improvement in system reliability;

- Redundancy in the monitoring system of the control valves in the inner tank pressure would significantly reduce the occurrence probability of the critical TOP event 1 ;

- A good control during the operation of tank filling and emptying is recommended in order to reduce the probability of occurrence of the critical TOP events 2 and 3;

- The failure of the Pressure Control Valves PCV9039 (i.e., the valves regulating the nitrogen purging into the annular space between the inner and outer vessels) greatly influences the occurrence of the critical TOP event 4.

An uncertainty analysis has been performed in order to assess the robustness of the results of the importance analyses. In fact, the importance measures depend on the values of the failure probabilities of the components of the storage system, which are uncertain. Thus, the results of the importance analysis need to be checked against the variability due to the uncertainty in the model input parameters. To do this, uncertainty in the occurrence probabilities of the most important basic events has been considered by uniform distributions with lower and upper bounds provided by SOL experts. The analysis has 
highlighted that the uncertainties in the occurrence probabilities of the basic events do not impact on the ranking of the most important causes identified for each TOP event: thus, the results discussed above can be considered robust.

\subsubsection{Uncertainty and sensitivity analysis}

As mentioned in Section 3.1.1, FTA has been performed to calculate the occurrence probabilities of the four critical events identified by the FMEA study.

In general, FTA allows resolving a TOP event into the combination of single primary events, which contribute to its realization. Then, the probability of the TOP event is computed as function of the probabilities of the basic events. However, in practice it is often difficult to evaluate the exact probability values of the primary events, which are affected by uncertainties. These latter need to be propagated through the Fault Tree model to assess the variability in the Top event failure probability due to the variability in the probability values of the basic events.

Two different analysis have been carried out, both based on Monte Carlo (MC) method:

- Sensitivity analysis to identifying the contribution of the various basic events to the uncertainty in the TOP event.

- Uncertainty analysis to propagate the uncertainties from the basic events up to the TOP events.

The results of the sensitivity analysis have shown that the estimations of the probabilities of the critical events are significantly influenced by the uncertainties in the failure probability values of the PSVs, human errors and uncontrolled filling and emptying operations. Then, improving the knowledge about the occurrence probability values of the identified events would reduce the uncertainty in the overall system reliability.

Once the most influent basic events have been identified, MC sampling technique has allowed to estimate the probability distribution expressing the variability in the probability of occurrence of every TOP event considered. Such distributions have shown that the values of the occurrence probability calculated without uncertainty, are always near to the values of the distributions associated to highest probability (distributions modes). However, the occurrence probability reference values are located toward the low values of the probability distributions. This finding entails that disregarding the uncertainty in the top event probability values does not lead to conservative results, and thus highlights the importance of giving due account to the uncertainty in reliability assessments.

\subsection{Structural analysis}

The Finite Element Method (FEM) has been used to perform the structural analysis to demonstrate the LBB criterion in both nominal and deviated conditions. FEM is a numerical technique for resolving complex problems, widely used in structural mechanics. The structural system to be studied is modeled by a set of finite elements interconnected at points, called nodes, that are organized in a mesh. The analysis provides the stresses and displacements, in each single element of the mesh. Therefore, the FEM methodology allows to identify the most stressed portions of the analyzed object.

For the case under study, the flat bottom tank has been modeled with a mesh of 262919 elements and 264267 nodes. The FEM analyses have been performed considering the tank behavior both in nominal operating conditions and in conditions of extreme loads, caused by the occurrence of the critical events identified by the previous failure analyses. The loads in ordinary conditions and the material strength have been set according to SOL internal documents.
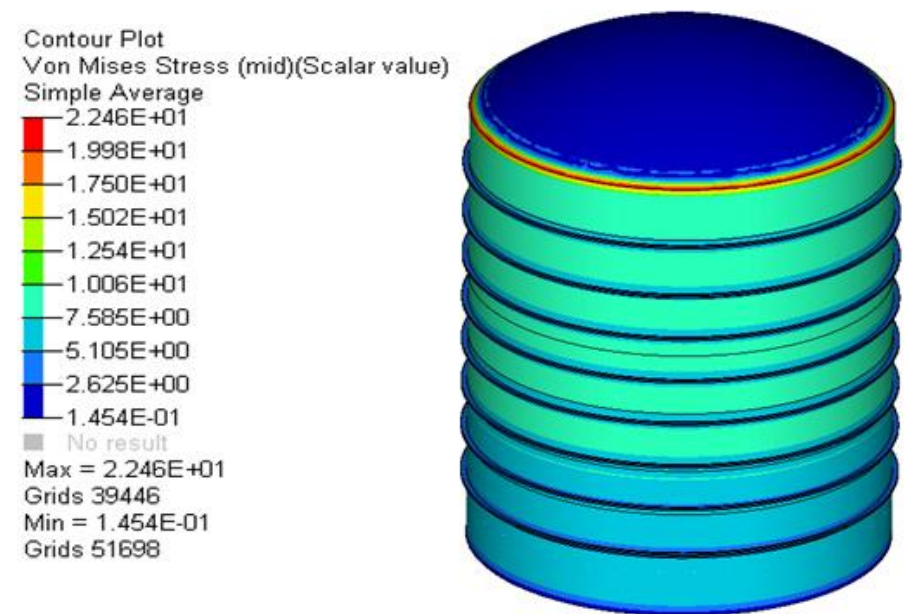

Figure 1: Von Mises stresses in the internal vessel under external ordinary pressure.

As expected, the analysis performed in ordinary conditions has shown that the tank is not subjected to excessive stresses and strains when everything operates normally: both Von Mises stresses and displacements are smaller than the maximum allowable limit set by the designers. Moreover, the analysis has highlighted the weakness of the structure in correspondence of the conjunction between the wall and the dome of the inner vessel. This is due to the fact that the stresses concentrated in this portion of the structure are very high, as exemplified in Figure 1 which shows the stress level in the inner tank under external pressure, in nominal conditions.

Analyses relevant to the critical scenarios have been carried out with the purpose of identifying the extreme values of pressure (TOP event 1,2 and 4) and liquid level (TOP event 3 ) that leads to the rup- 
ture of the tank structure. The resulting values are summarized in Table 5.

In particular, no value is reported for TOP event 3 , corresponding to the scenario of tank overfilling. Indeed, the analysis has shown that the criticality of such scenario is not due to the hydrostatic pressure of the liquid, but to the overpressure generated by the pumps that introduce the LOX in the vessel. Therefore, the TOP events 3 is analyzed similarly to TOP event 1 (i.e., overpressure in the inner tank).

Table 5. Extreme loads that lead to tank rupture.

\begin{tabular}{lll}
\hline & \multicolumn{1}{c}{ Parameter } & Max value \\
\cline { 2 - 3 } TOP event 1 & Pressure inner tank & $187.5 \mathrm{mbar}$ \\
TOP event 2 & Pressure inner tank & $-175 \mathrm{mbar}$ \\
TOP event 3 & Level in inner tank & ---------- \\
TOP event 4 & Pressure annular space & $1610 \mathrm{mbar}$ \\
\hline
\end{tabular}

The values listed in Table 5 are expected to be reached gradually, with a slow variation in the level and pressure values. This operating condition makes reasonable to assume that if cracks appear on the metal surfaces, then they slowly propagate. In these conditions, the detection of the leak is possible before the sudden rupture of the tank. In details, the analyses have highlighted that TOP events 1,2 and 3 lead to the rupture of the inner tank, whereas TOP event 4 generates failure on the outer casing. In the first case, the LOX penetrates into the annular space and ice forms on the outer casing; this can be visually detected. In the latter case, when the external vessel breaks, there is a release of perlite in the air, which, again, is easily detectable.

As in ordinary conditions, the loads corresponding to TOP events 1,2 and 3 show that the weak section of the storage system lies in correspondence of the conjunction between the wall and the dome of the inner vessel. TOP event 4 , instead, particularly stresses the surface of the vertical wall.

The results are compliant with the adopted manufacturing standards; in fact, quoting EIGA IGC Doc 127/13:

"The need to prevent a rupture by overpressure at the bottom of the inner tank is identified in the various available codes. API 650 talks of a frangible joint between the roof and the shell and gives it as a possible purchaser requirement. API 620, instead, establishes a link between the sizing of the anchorage straps and the design of the roof to shell junction. Finally, BS 7777 explicitly states that the weakest point of the structure shall not be at the bottom and present it as a further increase in safety".

\subsection{Conclusions}

The present study has been performed with the aim of evaluating the risks related to the catastrophic rupture of a flat bottom cryogenic tank containing liquefied gas. To do this, the cryogenic storage system has been deeply investigated in order to identify all the critical events that may evolve in dangerous scenarios affecting the structural safety of the cryogenic tank, together with their associated occurrence probabilities.

Four critical events have been identified, whose occurrence frequencies are very low. It has also emerged that regular inspections of PSVs yield a significant reduction in the occurrence frequencies of the top events.

The robustness of the risk analysis results has been checked by means of sensitivity and uncertainty analysis.

The structural behavior of the flat bottom tank has been analyzed both in ordinary operating conditions and in case of exceptional loads, due to the occurrence of the individuated TOP events, with the aim of assessing whether the identified scenarios produce loads that may lead to the rupture of the analyzed vessel.

As expected, the analysis performed in ordinary conditions has shown that the tank is not subjected to excessive stresses and strains. The analyses under the conditions brought by the critical events have allowed:

- Estimating the load conditions that lead to the rupture of the tank structure.

- Checking that there is a release of LOX or perlite when such load values are reached. This entails that both the failures of the inner and outer vessels can be detected.

- Verifying that an explosion of the tank can be regarded as improbable, due to the slow variation in level and pressure.

Finally, the structural analysis has also highlighted, in both ordinary and extreme conditions, the structure weakness in correspondence of the conjunction between the wall and the dome of the inner vessel, except for TOP event 4 . This is compliant with the recommendations of the design codes API 620, API 650 and BS 7777, which require to design the vessels in such a way to ensure that the weakest point of the inner tank is not at the bottom cylindrical seam, but at the top of the vessel (EIGA 2013).

\section{REFERENCES}

API 620. Design and Construction of Large, Welded, Lowpressure Storage Tank. American Petroleum Institute. API Publishing Services. Washington DC.

API 650. Welded Steel Tanks for Oil Storage. American Petroleum Institute. API Publishing Services. Washington DC.

Baraldi P., Compare M., Zio E. 2013. Component ranking by Birnbaum importance in presence of epistemic uncertainty in failure event probabilities. IEEE Transactions on Reliability 62(1): 37-48.

Baraldi, P., Zio, E., Compare, M. 2009. A method for ranking components importance in presence of epistemic uncertain- 
ties. Journal of Loss Prevention in the Process Industries 22(5):582-592.

BS 7777. Flat bottomed, vertical, cylindrical storage tank for low temperature service. British Standard Institute. London. United Kingdom.

BS 7910:2005. Guide to methods for assessing the acceptability of flaws in metallic structures. British Standard Institute. London. United Kingdom.

EIGA. 2013. IGC Doc 127/13/E. Bulk liquid oxygen, nitrogen and argon storage systems at production sites. Bruxelles.

Zio, E. 2009. Computational Methods for Reliability and Risk Analysis. Series in Quality, Reliability and Engineering Statistics Vol 14. World Scientific. Singapore.

Zio, E. 2007. An Introduction to the Basics of Reliability and Risk Analysis. Series in Quality, Reliability and Engineering Statistics Vol 13. World Scientific. Singapore. 